\title{
Differential Calculi on Quantum Spaces determined by Automorphisms
}

\author{
Aristophanes Dimakis \\ Department of Financial and Management Engineering \\ University of the Aegean, 31 Fostini Str., GR-82100 Chios, Greece \\ E-mail: dimakis@aegean.gr \\ Folkert Müller-Hoissen \\ Max-Planck-Institut für Strömungsforschung \\ Bunsenstrasse 10, D-37073 Göttingen, Germany \\ E-mail: Folkert.MuellerHoissen@mpi-sf.mpg.de
}

\begin{abstract}
If the bimodule of 1 -forms of a differential calculus over an associative algebra is the direct sum of 1-dimensional bimodules, a relation with automorphisms of the algebra shows up. This happens for some familiar quantum space calculi.
\end{abstract}

\section{Introduction}

A differential calculus over an associative algebra $\mathcal{A}$ is given by a graded associative algebra $\Omega(\mathcal{A})=\bigoplus_{r \geq 0} \Omega^{r}$, where $\Omega^{0}=\mathcal{A}$, together with a linear map d : $\Omega^{r} \rightarrow \Omega^{r+1}$ satisfying $\mathrm{d}^{2}=0$ and the graded Leibniz rule. Choosing generators $\theta^{s}, s \in S$, of the $\mathcal{A}$-bimodule $\Omega^{1}$ of 1-forms, the commutation relations with elements $a \in \mathcal{A}$ can be expressed as

$$
\theta^{s} a=\sum_{s^{\prime} \in S} \Phi(a)_{s^{\prime}}^{s} \theta^{s^{\prime}} \quad \forall a \in \mathcal{A}
$$

where $\Phi$ is a homomorphism from $\mathcal{A}$ into the $|S| \times|S|$-matrices with entries in $\mathcal{A}$. Suppose we can choose $\theta^{s}$ such that $\Phi$ becomes diagonal:

$$
\theta^{s} a=\phi_{s}(a) \theta^{s} \quad \forall s \in S
$$

Then the maps $\phi_{s}, s \in S$, have to be automorphisms of $\mathcal{A}$. In this case, $\Omega^{1}$ is the direct sum of 1-dimensional bimodules. If the special form (1.2) of the commutation relations can be achieved, calculations in the differential algebra are drastically simplified. Trivial examples are ordinary differential forms on a parallelizable manifold and Kähler differentials on freely 
generated commutative algebras. In these cases, we have $\phi_{s}=\mathrm{id}$, which means that the basis 1 -forms commute with all elements of $\mathcal{A}$. Surprisingly, this can also be achieved for certain differential calculi on noncommutative algebras [1, 2]. Simple examples with nontrivial automorphisms are given by $\mathrm{d} x x=(x+\ell) \mathrm{d} x$ [3, 4, 5] and $\mathrm{d} x x=q x \mathrm{~d} x$ [3, 6, 7] on the commutative algebra freely generated by $x$, setting $\theta^{1}=\mathrm{d} x$. A large class of examples arises from differential calculi on Cayley graphs ('group lattices') of a finite group $G$, where $S \subset G \backslash\{e\}$ and $\phi_{s}=R_{s}^{*}$, the pull-back with the right action of $s \in S$ on $G$ [8, 9]. There are more examples [10], some will be discussed in this work. Section 2 recalls a recipe [10] to construct differential calculi with the property (1.2). The two subsequent sections present some 'quantum space' examples. Section 5 shows how pseudo-Riemannian structures can be introduced on algebras with such a differential calculus.

\section{From automorphisms to differential calculi}

Let $\mathcal{A}$ be an associative algebra and $\left\{\phi_{s} \mid s \in S\right\}$ a finite set of automorphisms. Naturally associated with the latter are the discrete derivatives

$$
e_{s}=\phi_{s}-\mathrm{id}
$$

A generalization is given by twisted inner derivations:

$$
e_{s}=a_{s} \phi_{s}-R_{a_{s}}
$$

with special elements $a_{s} \in \mathcal{A}$ and the right action $R_{a}$ by $a$. As a consequence,

$$
e_{s}\left(a a^{\prime}\right)=e_{s}(a) \phi_{s}\left(a^{\prime}\right)+a e_{s}\left(a^{\prime}\right) \quad \forall a, a^{\prime} \in \mathcal{A}
$$

so that $e_{s}$ is a twisted derivation. Other twisted derivations are obtained from twisted inner derivations as limits

$$
e_{s}=\lim _{t \rightarrow 0} \frac{1}{t}\left(a_{s} \phi_{s}^{t}-R_{a_{s}}\right)
$$

where $\phi_{s}^{t}$ is a one-parameter group of automorphisms with $\phi_{s}^{0}=\mathrm{id}$.

The $\mathcal{A}$-bimodule $\mathbb{X}$ of 'vector fields' is generated by $\left\{e_{s}\right\}$ such that

$$
\left(e_{s} \cdot a\right) a^{\prime}:=e_{s}\left(a^{\prime}\right) a \quad a \cdot e_{s}:=e_{s} \cdot \phi_{s}(a) .
$$

In the following, we require that $\left\{e_{s} \mid s \in S\right\}$ is a left and right $\mathcal{A}$-module basis of $\mathbb{X}$. A dual $\mathcal{A}$-bimodule $\hat{\Omega}^{1}$ of ' 1 -forms' is then determined via

$$
\langle a \omega, X\rangle:=a\langle\omega, X\rangle, \quad\langle\omega, X \cdot a\rangle:=\langle\omega, X\rangle a, \quad\langle\omega a, X\rangle:=\langle\omega, a \cdot X\rangle
$$

for all $X \in \mathbb{X}, \omega \in \hat{\Omega}^{1}$. The basis $\left\{\theta^{s} \mid s \in S\right\}$ dual to $\left\{e_{s}\right\}$, i.e. $\left\langle\theta^{s}, e_{s^{\prime}}\right\rangle=\delta_{s^{\prime}}^{s}$, then satisfies (1.2). Now $\langle\mathrm{d} a, X\rangle:=X a$ determines a linear map $\mathrm{d}: \mathcal{A} \rightarrow \hat{\Omega}^{1}$ such that

$$
\mathrm{d} a=\sum_{s \in S} e_{s}(a) \theta^{s}
$$


The map d satisfies the Leibniz rule $\mathrm{d}\left(a a^{\prime}\right)=(\mathrm{d} a) a^{\prime}+a\left(\mathrm{~d} a^{\prime}\right)$ if $e_{s}, s \in S$, are twisted derivations. Furthermore, $\left(\hat{\Omega}^{1}, \mathrm{~d}\right)$ is inner:

$$
\mathrm{d} a=[\vartheta, a] \quad \text { with } \quad \vartheta:=\sum a_{s} \theta^{s} .
$$

Remark. In general, $\hat{\Omega}^{1}$ is larger than the $\mathcal{A}$-bimodule $\Omega^{1}$ generated by $\mathrm{d} \mathcal{A}$, since $\theta^{s}$ need not be of the form $\sum_{i} a_{i} \mathrm{~d} a_{i}^{\prime}$. If there are 'coordinates' $x^{s} \in \mathcal{A}$ s.t. $e_{s}\left(x^{s^{\prime}}\right)$ are the components of an invertible matrix, then $\theta^{s} \in \Omega^{1}$ and the two spaces coincide. In most cases, however, $\mathcal{A}$ can be appended by additional relations (typically: inverses of some elements), such that $\hat{\Omega}^{1}(\hat{\mathcal{A}})=\Omega^{1}(\hat{\mathcal{A}})$ for the new algebra $\hat{\mathcal{A}}$.

The first order differential calculus extends to higher orders such that

$$
\mathrm{d} \omega=[\vartheta, \omega]_{\operatorname{grad}}-\Xi(\omega)
$$

involving the graded commutator. The map $\Xi$ has to satisfy

$$
\Xi\left(a \omega a^{\prime}\right)=a \Xi(\omega) a^{\prime}, \quad \Xi\left(\omega \omega^{\prime}\right)=\Xi(\omega) \omega^{\prime}+(-1)^{\operatorname{grad}(\omega)} \omega \Xi\left(\omega^{\prime}\right) .
$$

\section{WZ calculus on the quantum plane}

The $q$-plane (see [11, for example) is the algebra generated by two elements $x, y$ subject to $x y=q y x$ with $q \in \mathbb{C} \backslash\{0, \pm 1\}$. Let us choose the special automorphisms

$$
\phi_{1}(x)=r^{-1} x, \quad \phi_{1}(y)=r^{-1} y, \quad \phi_{2}(x)=x, \quad \phi_{2}(y)=r^{-1} y
$$

with $r \notin\{0,1\}$. Applying the construction of the preceding section, starting with the discrete derivatives $e_{s}:=\phi_{s}-\mathrm{id}$, leads to

$$
\theta^{1}=\frac{1}{1-r} \mathrm{~d} x x^{-1}, \quad \theta^{2}=\frac{1}{1-r}\left(\mathrm{~d} y y^{-1}-\mathrm{d} x x^{-1}\right)
$$

where we need inverses of $x$ and $y$, thus passing over to the quantum torus. From (1.2) we recover the Wess-Zumino calculus [12] on the $q$-plane:

$$
\begin{array}{ll}
x \mathrm{~d} x=p q \mathrm{~d} x x & y \mathrm{~d} x=p \mathrm{~d} x y \\
y \mathrm{~d} y=p q \mathrm{~d} y y & x \mathrm{~d} y=q \mathrm{~d} y x+(p q-1) \mathrm{d} x y
\end{array}
$$

where $p:=r / q$. Furthermore, $\theta^{1} \theta^{2}=-\theta^{2} \theta^{1},\left(\theta^{1}\right)^{2}=0=\left(\theta^{2}\right)^{2}, \mathrm{~d} \omega=[\vartheta, \omega]_{\operatorname{grad}}$ (so that $\Xi=0)$ with $\vartheta=\theta^{1}+\theta^{2}$, and $\mathrm{d} \vartheta=0$.

\section{Bicovariant differential calculus on the quantum group $G L_{p, q}(2)$}

The matrix quantum group $G L_{p, q}(2)$ is the algebra $\mathcal{A}$ generated by elements $a, b, c, d$ subject to the relations

$$
\begin{array}{lll}
a b=p b a & a c=q c a & b c=\frac{q}{p} c b \\
b d=q d b & c d=p d c & a d=d a+\left(p-\frac{1}{q}\right) b c
\end{array}
$$


and supplied with a Hopf algebra structure [11. Its automorphisms are given by scalings $(a, b, c, d) \mapsto(\alpha a, \beta b, \gamma c, \delta d)$ with constants satisfying $\alpha \delta=\beta \gamma \neq 0$. The relations defining a bicovariant differential calculus on a matrix quantum group are usually expressed in terms of left-coinvariant Maurer-Cartan 1-forms:

$$
\left(\begin{array}{ll}
\vartheta^{1} & \vartheta^{2} \\
\vartheta^{3} & \vartheta^{4}
\end{array}\right):=\mathcal{S}\left(\begin{array}{ll}
a & b \\
c & d
\end{array}\right) \mathrm{d}\left(\begin{array}{ll}
a & b \\
c & d
\end{array}\right)
$$

using the antipode $\mathcal{S}$ (see [13, 14] for details). Combined with (2.7), this implies

$$
\left(\begin{array}{l}
\vartheta^{1} \\
\vartheta^{3}
\end{array}\right)=\mathcal{S}\left(\begin{array}{ll}
a & b \\
c & d
\end{array}\right) \sum_{s=1}^{4}\left(\begin{array}{l}
e_{s}(a) \\
e_{s}(c)
\end{array}\right) \theta^{s}
$$

and a corresponding formula with $\vartheta^{1}, \vartheta^{3}, e_{s}(a), e_{s}(c)$ replaced by $\vartheta^{2}, \vartheta^{4}, e_{s}(b), e_{s}(d)$, respectively. There are two distinguished bicovariant calculi on $G L_{p, q}(2)$ [15, 13]. For one of them, 1-forms satisfying (1.2) have been derived in [10]. The other is given by

$$
\begin{aligned}
& \vartheta^{1} a=\frac{1}{r} a \vartheta^{1}, \quad \vartheta^{1} b=b \vartheta^{1}, \quad \vartheta^{2} a=-\left(q-\frac{1}{p}\right) b \vartheta^{1}+\frac{1}{p} a \vartheta^{2} \\
& \vartheta^{2} b=\frac{1}{p} b \vartheta^{2}, \quad \vartheta^{3} a=\frac{1}{q} a \vartheta^{3}, \quad \vartheta^{3} b=-\left(p-\frac{1}{q}\right) a \vartheta^{1}+\frac{1}{q} b \vartheta^{3} \\
& \vartheta^{4} a=a\left[\frac{1}{r}(1-r)^{2} \vartheta^{1}+\vartheta^{4}\right]+\frac{1-r}{r} b \vartheta^{3}, \quad \vartheta^{4} b=\frac{1-r}{r} a \vartheta^{2}+\frac{1}{r} b \vartheta^{4}
\end{aligned}
$$

where the remaining relations are obtained from the above by replacing $a$ by $c$ and $b$ by $d$ (see example 2 in section 6 of [13]). A lengthy calculation yields the 1 -forms

$$
\begin{aligned}
& \tilde{\theta}^{1}=\vartheta^{1}, \quad \tilde{\theta}^{2}=-r d \vartheta^{1}+c \vartheta^{2}, \quad \tilde{\theta}^{3}=a \vartheta^{1}+b \vartheta^{3} \\
& \tilde{\theta}^{4}=-p d a \vartheta^{1}+c a \vartheta^{2}-(p / q) b d \vartheta^{3}+(p / q) b c \vartheta^{4}
\end{aligned}
$$

which satisfy (1.2) with the automorphisms determined by

$$
\begin{aligned}
& \tilde{\phi}_{1}(a, b, c, d)=\left(r^{-1} a, b, r^{-1} c, d\right), \quad \tilde{\phi}_{2}(a, b, c, d)=\left(r^{-1} a, q^{-1} b, p^{-1} c, d\right) \\
& \tilde{\phi}_{3}(a, b, c, d)=\left(r^{-1} a, q^{-1} b, p^{-1} c, d\right), \quad \tilde{\phi}_{4}(a, b, c, d)=\left(r^{-1} a, q^{-2} b, q p^{-1} c, d\right) \text {. }
\end{aligned}
$$

Multiplying the 1-forms $\tilde{\theta}^{s}$ by non-zero constants, arbitrary positive integer powers of $b, c$, and arbitrary integer powers of the quantum determinant $\mathcal{D}:=a d-p b c$, preserves the property (1.2), but with different automorphisms. In this way we can achieve $\tilde{\phi}_{2} \neq \tilde{\phi}_{3}$, for example. (4.4) can only be solved for the Maurer-Cartan 1-forms if we pass over to the algebra $\hat{\mathcal{A}}$ obtained from $\mathcal{A}$ by requiring $b$ and $c$ to have inverses. Comparing the resulting expressions with (4.3) then shows that $e_{s}, s \in S$, do not have values in $\mathcal{A}$, but rather in $\hat{\mathcal{A}}$. Let us write

$$
\tilde{\theta}^{s}=b c \theta^{s}
$$

with new (generalized) 1-forms $\theta^{s}$. The corresponding automorphisms are given by

$$
\begin{array}{ll}
\phi_{1}(a, b, c, d)=\left(a, q p^{-1} b, q^{-2} c, r^{-1} d\right), & \phi_{2}(a, b, c, d)=\left(a, p^{-1} b, q^{-1} c, r^{-1} d\right) \\
\phi_{3}(a, b, c, d)=\left(a, p^{-1} b, q^{-1} c, r^{-1} d\right), & \phi_{4}(a, b, c, d)=\left(a, r^{-1} b, c, r^{-1} d\right)
\end{array}
$$


and (4.4) leads to

$$
\begin{aligned}
& \vartheta^{1}=b c \theta^{1}, \quad \vartheta^{2}=\frac{q}{p} b\left(d \theta^{1}+\theta^{2}\right), \quad \vartheta^{3}=-p a c \theta^{1}+c \theta^{3}, \\
& \vartheta^{4}=-q a d \theta^{1}-q a \theta^{2}+\frac{1}{p} d \theta^{3}+\frac{q}{p} \theta^{4}
\end{aligned}
$$

The calculus is inner with (see [13])

$$
\vartheta=\frac{r}{1-r}\left(r \vartheta^{1}+\vartheta^{4}\right)=\frac{q}{1-r}\left(-r \mathcal{D} \theta^{1}-r a \theta^{2}+d \theta^{3}+q \theta^{4}\right) .
$$

Comparison with (2.8) shows that $a_{1}=-(q r / 1-r) \mathcal{D}, a_{2}=-(q r / 1-r) a, a_{3}=(q / 1-r) d$ and $a_{4}=\left(q^{2} / 1-r\right) \mathbf{1}$ (where $\mathbf{1}$ is the unit in $\mathcal{A}$ ). This determines the twisted inner derivations (2.2). By rescalings of the $\theta^{s}$ we can achieve somewhat simpler expressions. Assuming again $b$ and $c$ to be invertible, the Maurer-Cartan 2-form relations (see [13]) translate to the considerably simpler relations

$$
\begin{aligned}
& \left(\theta^{1}\right)^{2}=\left(\theta^{2}\right)^{2}=\left(\theta^{3}\right)^{2}=\left(\theta^{4}\right)^{2}=0, \\
& \theta^{2} \theta^{1}=-r \theta^{1} \theta^{2}, \quad \theta^{3} \theta^{1}=-\theta^{1} \theta^{3}, \quad \theta^{3} \theta^{2}=-\theta^{2} \theta^{3}, \\
& \theta^{4} \theta^{1}=-r \theta^{1} \theta^{4}+\left(p-\frac{1}{q}\right) \theta^{2} \theta^{3}, \quad \theta^{4} \theta^{2}=-r \theta^{2} \theta^{4}, \quad \theta^{4} \theta^{3}=-r \theta^{3} \theta^{4} .
\end{aligned}
$$

The full differential calculus is inner, i.e. $\Xi=0$.

It is quite surprising that for bicovariant calculi on $G L_{p, q}(2)$, with the extension of the algebra by inverses of $b$ and $c$, the bimodule of 1 -forms splits into a direct sum of 1-dimensional bimodules, so that we have the 'automorphism structure' (1.2). We do not know yet whether this result extends to bicovariant calculi on other matrix quantum groups (see [11, for example). ${ }^{1}$ Is there a deeper relation with bicovariance? Let $\vartheta^{s}$ be the corresponding leftinvariant Maurer-Cartan forms, so that $\Delta_{L} \vartheta^{s}=1 \otimes \vartheta^{s}$ with the left-coaction $\Delta_{L}$. Writing $\theta^{s}=\sum_{s^{\prime} \in S} \hat{a}_{s^{\prime}}^{s} \vartheta^{s^{\prime}}$, we find

$$
\sum_{s^{\prime} \in S} \Delta\left(\hat{a}_{s^{\prime}}^{s}\right) 1 \otimes \vartheta^{s^{\prime}} \Delta(a)=\Delta_{L}\left(\theta^{s}\right) \Delta(a)=\Delta_{L}\left(\theta^{s} a\right)
$$

where $\Delta$ is the coproduct. For all $a \in \mathcal{A}$, this has to be equal to

$$
\Delta\left(\phi_{s} a\right) \Delta_{L}\left(\theta^{s}\right)=\Delta\left(\phi_{s} a\right) \sum_{s^{\prime} \in S} \Delta\left(\hat{a}_{s^{\prime}}^{s}\right) 1 \otimes \vartheta^{s^{\prime}}
$$

Additional conditions arise from right-covariance of the calculus. Together with the knowledge of the corresponding automorphism groups, these conditions restrict the elements $\hat{a}_{s^{\prime}}$ and should lead to the possible choices of 1 -forms $\theta^{s}$ or corresponding obstructions. This program has still to be carried out.

\footnotetext{
${ }^{1}$ Even for $G L_{p, q}(2)$ we do not have the full answer yet, since there is a 1-parameter family of bicovariant calculi [13, 14] and we were only able so far to treat special members.
} 


\section{Differential geometry in terms of the basis $\theta^{s}$}

An automorphism $\phi$ of $\mathcal{A}$ is called differentiable with respect to a differential calculus $(\hat{\Omega}(\mathcal{A}), \mathrm{d})$ if it extends to a homomorphism of $\hat{\Omega}(\mathcal{A})$ such that $\phi \circ \mathrm{d}=\mathrm{d} \circ \phi$. In the above examples, the $\phi_{s}$ are indeed differentiable. For the $q$-plane WZ-calculus, for example, one finds $\phi_{s} \theta^{s^{\prime}}=\theta^{s^{\prime}}$. Let us now assume that $\left\{\phi_{s}, \phi_{s}^{-1} \mid s \in S\right\}$ are differentiable. Then we can introduce a left $\mathcal{A}$-linear tensor product basis:

$$
\theta^{s} \otimes_{L} \theta^{s^{\prime}}:=\theta^{s} \otimes_{\mathcal{A}} \phi_{s}^{-1} \theta^{s^{\prime}} \Longrightarrow a \theta^{s} \otimes_{L}\left(a^{\prime} \theta^{s^{\prime}}\right)=a a^{\prime} \theta^{s} \otimes_{L} \theta^{s^{\prime}} .
$$

In particular, a metric should then be an element ${ }^{2}$

$$
\mathbf{g}=\sum_{s, s^{\prime} \in S} g_{s, s^{\prime}} \theta^{s} \otimes_{L} \theta^{s^{\prime}}
$$

with $g_{s, s^{\prime}} \in \mathcal{A}$. Let $\nabla$ be a linear connection, i.e. a connection on $\hat{\Omega}^{1}$. Then

$$
\nabla \omega=\vartheta \otimes_{\mathcal{A}} \omega-\sum_{s^{\prime} \in S} \theta^{s^{\prime}} \otimes_{\mathcal{A}} \mathcal{V}_{s^{\prime}} \omega \quad \forall \omega \in \hat{\Omega}^{1}
$$

with 'parallel transport operators' $\mathcal{V}_{s}$ satisfying

$$
\mathcal{V}_{s}(a \omega)=\left(\phi_{s}^{-1} a\right) \mathcal{V}_{s} \omega
$$

Furthermore,

$$
\mathcal{V}_{s}\left(\omega \otimes_{L} \omega^{\prime}\right):=\left(\mathcal{V}_{s} \omega\right) \otimes_{L}\left(\mathcal{V}_{s} \omega^{\prime}\right) \quad \forall \omega, \omega^{\prime} \in \hat{\Omega}^{1}
$$

determines a connection $\nabla$ on $\hat{\Omega}^{1} \otimes_{L} \hat{\Omega}^{1}$. So we can define metric compatibility:

$$
\nabla \mathrm{g}=0 \quad \Longleftrightarrow \quad \mathcal{V}_{s}(\mathrm{~g})=\mathrm{g} \quad \forall s \in S
$$

A torsion-free metric-compatible linear connection should be called a Levi-Civita connection. For differential calculi associated with (a subclass of) Cayley graphs ('group lattices') of finite groups, the corresponding generalized (pseudo-) Riemannian geometry has been developed in [8, 9]. This includes (pseudo-) Riemannian geometry of hyper-cubic lattices as a special case. The above scheme can be applied as well to introduce analogs of (pseudo-) Riemannian structures on quantum planes and quantum groups.

\section{References}

[1] A. Dimakis and F. Müller-Hoissen: Int. J. Mod. Phys. A (Proc. Suppl.) 3A (1993) 214.

[2] A. Dimakis and J. Madore: J. Math. Phys. 37 (1996) 4647.

[3] A. Dimakis and F. Müller-Hoissen: Phys. Lett. B 295 (1992) 242.

\footnotetext{
${ }^{2}$ See [9] for arguments why a metric should be defined in this way rather than as an element of $\hat{\Omega}^{1} \otimes_{\mathcal{A}} \hat{\Omega}^{1}$.
} 
[4] A. Dimakis, F. Müller-Hoissen, and T. Striker: J. Phys. A: Math. Gen. 26 (1992) 1927.

[5] A. Dimakis, F. Müller-Hoissen, and T. Striker: Phys. Lett. B 300 (1993) 141.

[6] Yu. I. Manin: Theor. Math. Phys. 92 (1992) 997.

[7] M. Karoubi: Contemp. Math. 279 (2001) 177.

[8] A. Dimakis and F. Müller-Hoissen: J. Math. Phys. 44 (2003) 1781.

[9] A. Dimakis and F. Müller-Hoissen: J. Math. Phys. 44 (2003) 4220.

[10] A. Dimakis and F. Müller-Hoissen: J. Phys. A: Math. Gen. 37 (2004) 2307.

[11] A. Klimyk and K. Schmüdgen: Quantum Groups and their Representations. Springer, Berlin, 1997.

[12] J. Wess and B. Zumino: Nucl. Phys. B (Proc. Suppl.) 18 (1990) 302.

[13] F. Müller-Hoissen: J. Phys. A: Math. Gen. 25 (1992) 1703.

[14] F. Müller-Hoissen and C. Reuten: J. Phys. A: Math. Gen. 26 (1993) 2955.

[15] G. Maltsiniotis: C. R. Acad. Sci. Paris 311 (1990) 831. 